\title{
INVESTIGATION OF G-NETWORK WITH BYPASSES OF QUEUEING SYSTEMS BY POSITIVE CUSTOMERS AT A NON-STATIONARY REGIME
}

\author{
Victor Naumenko ${ }^{1}$, Dmitry Kopats ${ }^{1}$, Mikhail Matalytski ${ }^{2}$, Andrey Pankov ${ }^{1}$ \\ ${ }^{I}$ Faculty of Mathematics and Computer Science, Yanka Kupala State University of Grodno \\ Grodno, Belarus \\ ${ }^{2}$ Department of Mathematics, Czestochowa University of Technology \\ Czestochowa, Poland \\ victornn86@gmail.com
}

Received: 22 October 2019; Accepted: 27 April 2020

\begin{abstract}
An open queuing network (QN) with single-line queuing systems (QS) is considered. QS are characterized by the presence of bypasses and the possibility of coming of negative customers. The network receives two independent elementary streams. The first stream is formed from the ordinary (positive) customers, while the second is composed of negative customers. Arriving of each negative customer to the system destroys exactly one positive customer in the queue, if those are contained. Negative customers do not require maintenance as the service of positive customers in the network systems is carried out in accordance with the FIFO discipline. Positive customers with a probability depending on the state of the node when they are sent to it are added to the queue, and with an additional probability, they immediately bypass it and behave in the future as served. The service in the systems is exponential, the routing of positive customers in the network is Markov, taking into account the possibility of turning the customer into a negative one after sending it to another system.
\end{abstract}

MSC 2010: 60K20, 60K25, 68M20, $90 B 22$

Keywords: queuing network, non-stationary regime, negative customers, bypass

\section{Introduction}

QN with negative customers has introduced by E. Gelenbe [1, 2]. The main application of this model is to simulate the effects of computer viruses on executable programs on a server or on user's local computer. In transition mode, this network has been investigated in [3].

QN with bypasses of nodes have introduced in [4]. It is shown in this work that such model includes the possibility to bypass systems due to restrictions of the number of customers or of the estimated waiting time. Stationary probabilities of network states in the form of a product have found. In transition mode, a network 
with bypasses has been investigated in [5]. The use of a network with bypasses is associated with the possibility of a client arriving at a service center of an information network not to join the queue for one reason or another, but has to switch to another service center.

In this paper, we consider an open QN with negative customers and bypasses of nodes with positive customers taking into account the first two features, and bypassing service systems is carried out only by positive customers. In the stationary mode, they were investigated in $[6,7]$. In [8], the expected revenues of the systems of this network have been found when the revenues from transitions are random variables with known moments of the first two orders.

Consider an open G-network with $n$ single-queues QS. The network state at time $t$ is described by the vector of dimension $n+1: \vec{k}=\vec{k}(t)=(\vec{k}, t)=$ $=\left(k_{1}, k_{2}, \ldots, k_{n}, t\right)$, which forms a homogeneous Markov process with a countable count of states, where the state $\left(k_{i}, t\right)$ means that at time $t$ in QS $S_{i}$, there are $k_{i}$ positive customers, $i=\overline{1, n}$.

An independent Poisson flow of positive customers with rate $\lambda_{0 i}^{+}$and a Poisson flow of negative customers with rate $\lambda_{0 i}^{-}$arrive to QS $S_{i}$ from outside (system $S_{0}$ ), $i=\overline{1, n}$. All customer flows arriving to QS are assumed to be independent. A positive customer arriving to the system increases the count of customers in the system by unity and requires service. Requests are serviced in the order received. The service time of customers in the $i$-th QS is independent, and does not depend on durations of service of positive customers which are an exponential distribution with the rate $\mu_{i}, i=\overline{1, n}$.

The positive customer is sent to the $i$-th QS from the external environment or from other systems, when the network is in a state $\vec{k}$, with a probability $f^{(i)}\left(k_{i}\right)$, where $k_{i}$ - number of positive customers in the $i$-th QS, joins the queue, but with an additional probability $1-f^{(i)}\left(k_{i}\right)$ does not join the queue, considered instantly served (i.e., bypasses the QS).

The positive customer gets serviced in $S_{i}$ with probability $p_{i j}^{+}$, moves to QS $S_{j}$ as a positive customer and with probability $p_{i j}^{-}$- as a negative customer and with probability $p_{i 0}=1-\sum_{j=1}^{n}\left(p_{i j}^{+}+p_{i j}^{-}\right)$comes out of the network to the external environment, $i, j=\overline{1, n}$.

Negative customers represent a special type of customers; they are not serviced and arrive directly in the QS (for them $f^{(i)}\left(k_{i}\right)=1$ ), where they reduce the queue length by one if the number of customers in the system is greater than zero, and do not make any changes if there are no customers in the QS. After these operations, negative customers disappear and subsequently do not affect the network. 
Let $\phi_{i}(\vec{k})$ - be the conditional probability that the customer arriving at the $i$-th QS, when the network is in state $\vec{k}$, will not be served by any of the QS and will not change the status of the network;

$\psi_{i j}(\vec{k})$ - the conditional probability that the customer arriving at the $i$-th QS, when the network is in state $\vec{k}$, for the first time will receive a servicing in the $j$-th QS, $j=\overline{1, n}$;

$\xi_{i j}(\vec{k})$ - the conditional probability that the customer, arrived from the outside at the $i$-th QS, when the network is in state $\vec{k}$, for the first time will have an impact on the $j$-th QS as a negative customer, $j=\overline{1, n}$;

$\alpha_{i}(\vec{k})$ - the conditional probability that the customer, whose servicing in the $i$-th QS is completed, when the network is in state $\vec{k}$, will no longer be served by any of the QS and will leave the network;

$\beta_{i j}(\vec{k})$ - the conditional probability that the customer, whose servicing in the $i$-th QS was completed, when the network is in state $\vec{k}$, for the first time after that to get service in the $j$-th QS, $i, j=\overline{1, n}$;

$\gamma_{i j}(\vec{k})$ - the conditional probability that the customer, has served in the $i$-th QS, when the network is in state $\vec{k}$ for the first time will have an impact on the $j$-th QS, while being a negative customer, $i, j=\overline{1, n}$.

According to the formula of total probability, we obtain:

$$
\begin{gathered}
\phi_{i}(\vec{k})=\left(1-f^{(i)}(\vec{k})\right)\left(p_{i 0}+\sum_{j=1}^{n}\left[p_{i j}^{+} \phi_{j}(\vec{k})+p_{i j}^{-}\left(1-u\left(k_{j}\right)\right)\right], i=\overline{1, n}\right. \\
\psi_{i j}(\vec{k})=f^{(i)}(\vec{k}) \delta_{i j}+\left(1-f^{(i)}(\vec{k})\right) \sum_{l=1}^{n} p_{i l}^{+} \psi_{l j}(\vec{k}), i, j=\overline{1, n} \\
\xi_{i j}(\vec{k})=\left(1-f^{(i)}(\vec{k})\right)\left[p_{i j}^{-}+\sum_{l=1}^{n} p_{i l}^{+} \xi_{l j}(\vec{k})\right], i, j=\overline{1, n} \\
\alpha_{i}(\vec{k})=p_{i 0}+\sum_{j=1}^{n}\left[p_{i j}^{+} \phi_{j}\left(\vec{k}-I_{i}\right)+p_{i j}^{-}\left(\left(1-u\left(k_{j}\right)\right)+\delta_{i j}\left(2-u\left(k_{i}\right)\right)\right)\right], i=\overline{1, n} \\
\beta_{i j}(\vec{k})=\sum_{l=1}^{n} p_{i l}^{+} \psi_{l j}\left(\vec{k}-I_{i}\right), i, j=\overline{1, n}
\end{gathered}
$$




$$
\gamma_{i j}(\vec{k})=p_{i j}^{-}+\sum_{l=1}^{n} p_{i l}^{+} \xi_{l j}\left(\vec{k}-I_{i}\right), i, j=\overline{1, n}
$$

where: $\delta_{i j}=\left\{\begin{array}{l}1, i=j, \\ 0, i \neq j\end{array}\right.$ - the Kronecker delta, $I_{i}$ - vector, which is $\mathrm{i}$-th component equal to 1 , all the others are $0, i=\overline{1, n}, u(x)$ - Heaviside function, $u(x)=\left\{\begin{array}{l}1, x>0 \\ 0, x \leq 0\end{array}\right.$

The aim of the research is to derive the Kolmogorov system of difference-differential equations (DDE) for finding the non-stationary state probabilities and propose a method for solving it.

\section{The Kolmogorov system of difference-differential equations for the state probabilities}

Let $P(\vec{k}, t)$ - be the state probability of the network $\vec{k}$ at time $t$.

Lemma. Nonstationary state probabilities of the considered QN satisfy the Kolmogorov DDE system:

$$
\begin{aligned}
\frac{d P(\vec{k}, t)}{d t} & =-\sum_{i=1}^{n}\left[\lambda_{0 i}^{+}\left(1-\phi_{i}(\vec{k}) u\left(k_{i}\right)\right)+\mu_{i} u\left(k_{i}\right)\left(1-\beta_{i i}(\vec{k})\right)+\lambda_{0 i}^{-} u\left(k_{i}\right)\right] P(\vec{k}, t)+ \\
& +\sum_{i=1}^{n} \lambda_{0 i}^{+} \sum_{j=1}^{n} \psi_{j i}\left(\vec{k}-I_{i}\right) u\left(k_{i}\right) P\left(\vec{k}-I_{i}, t\right)+ \\
& +\sum_{i=1}^{n}\left[\lambda_{0 i}^{-}+\sum_{j=1}^{n} \lambda_{0 j}^{+} \xi_{j i}\left(\vec{k}+I_{i}\right)+\mu_{i} \alpha_{i}\left(\vec{k}+I_{i}\right)\right] P\left(\vec{k}+I_{i}, t\right)+ \\
& +\sum_{i=1}^{n} \sum_{\substack{j=1 \\
j \neq i}}^{n} \mu_{j} \beta_{j i}\left(\vec{k}+I_{j}-I_{i}\right) u\left(k_{i}\right) P\left(\vec{k}+I_{j}-I_{i}, t\right)+ \\
& +\sum_{i=1}^{n} \sum_{j=1}^{n} \mu_{j} \gamma_{j i}\left(\vec{k}+I_{j}+I_{i}\right) P\left(\vec{k}+I_{j}+I_{i}, t\right) .
\end{aligned}
$$

Proof. The possible transitions of our Markov process in the state $(\vec{k}, t)$ during time $\Delta t$ :

1) from the state $\left(\vec{k}-I_{i}, t\right)$ with probability

$$
\lambda_{0 i}^{+} \psi_{j i}\left(\vec{k}-I_{i}\right) u\left(k_{i}\right) \Delta t+o(\Delta t), i, j=\overline{1, n}
$$


2) from the state $\left(\vec{k}+I_{i}, t\right)$ with probability

$$
\left[\lambda_{0 i}^{-}+\lambda_{0 j}^{+} \xi_{j i}\left(\vec{k}+I_{i}\right)+\mu_{i} \alpha_{i}\left(\vec{k}+I_{i}\right)\right] \Delta t+o(\Delta t), i, j=\overline{1, n} ; 1, n
$$

3) from the state $\left(\vec{k}+I_{i}-I_{j}, t\right)$ with probability

$$
\mu_{j} \beta_{j i}\left(\vec{k}+I_{j}-I_{i}\right) u\left(k_{i}\right) \Delta t+o(\Delta t), i, j=\overline{1, n}
$$

4) from the state $\left(\vec{k}+I_{i}+I_{j}, t\right)$ with probability

$$
\mu_{j} \gamma_{j i}\left(\vec{k}+I_{j}+I_{i}\right) \Delta t+o(\Delta t), i, j=\overline{1, n}
$$

5) from the state $(\vec{k}, t)$ with probability

$$
1-\left[\lambda_{0 i}^{+}\left(1-\phi_{i}(\vec{k}) u\left(k_{i}\right)\right)+\mu_{i} u\left(k_{i}\right)\left(1-\beta_{i i}(\vec{k})\right)+\lambda_{0 i}^{-} u\left(k_{i}\right)\right] \Delta t+o(\Delta t)
$$

6) from other states with probability $o(\Delta t)$.

Then, using the formula of total probability, we can obtain

$$
\begin{aligned}
P(\vec{k}, t & +\Delta t)=\sum_{i=1}^{n} \lambda_{0 i}^{+} \sum_{j=1}^{n} \psi_{j i}\left(\vec{k}-I_{i}\right) u\left(k_{i}\right) P\left(\vec{k}-I_{i}, t\right) \Delta t+ \\
& +\sum_{i=1}^{n}\left[\lambda_{0 i}^{-}+\sum_{j=1}^{n} \lambda_{0 j}^{+} \xi_{j i}\left(\vec{k}+I_{i}\right)+\mu_{i} \alpha_{i}\left(\vec{k}+I_{i}\right)\right] P\left(\vec{k}+I_{i}, t\right) \Delta t+ \\
& +\sum_{i=1}^{n} \sum_{\substack{j=1 \\
j \neq i}}^{n} \mu_{j} \beta_{j i}\left(\vec{k}+I_{j}-I_{i}\right) u\left(k_{i}\right) P\left(\vec{k}+I_{j}-I_{i}, t\right) \Delta t+ \\
& +\sum_{i=1}^{n} \sum_{j=1}^{n} \mu_{j} \gamma_{j i}\left(\vec{k}+I_{j}+I_{i}\right) P\left(\vec{k}+I_{j}+I_{i}, t\right) \Delta t \\
& +P(\vec{k}, t)\left\{1-\left[\lambda_{0 i}^{+}\left(1-\phi_{i}(\vec{k}) u\left(k_{i}\right)\right)+\mu_{i} u\left(k_{i}\right)\left(1-\beta_{i i}(\vec{k})\right)+\lambda_{0 i}^{-} u\left(k_{i}\right)\right] \Delta t\right\}+o(\Delta t) .
\end{aligned}
$$

Dividing both sides of this relationship by $\Delta t$ and taking the limit $\Delta t \rightarrow 0$, we obtain a system of equations for the state probabilities of the network (7). 


\section{Finding the state probabilities of the network operating in saturation mode}

We assume that all QS networks operate in saturation mode, i.e. $k_{i}(t)>0$ $\forall t>0, i=\overline{1, n}$, then system (7) takes the form

$$
\begin{aligned}
\frac{d P(\vec{k}, t)}{d t} & =-\sum_{i=1}^{n}\left[\lambda_{0 i}^{+}\left(1-\phi_{i}(\vec{k})\right)+\mu_{i}\left(1-\beta_{i i}(\vec{k})\right)+\lambda_{0 i}^{-}\right] P(\vec{k}, t)+ \\
& +\sum_{i=1}^{n} \lambda_{0 i}^{+} \sum_{j=1}^{n} \psi_{j i}\left(\vec{k}-I_{i}\right) P\left(\vec{k}-I_{i}, t\right)+ \\
& +\sum_{i=1}^{n}\left[\lambda_{0 i}^{-}+\sum_{j=1}^{n} \lambda_{0 j}^{+} \xi_{j i}\left(\vec{k}+I_{i}\right)+\mu_{i} \alpha_{i}\left(\vec{k}+I_{i}\right)\right] P\left(\vec{k}+I_{i}, t\right)+ \\
& +\sum_{i=1}^{n} \sum_{\substack{j=1 \\
j \neq i}}^{n} \mu_{j} \beta_{j i}\left(\vec{k}+I_{j}-I_{i}\right) P\left(\vec{k}+I_{j}-I_{i}, t\right)+ \\
& +\sum_{i=1}^{n} \sum_{j=1}^{n} \mu_{j} \gamma_{j i}\left(\vec{k}+I_{j}+I_{i}\right) P\left(\vec{k}+I_{j}+I_{i}, t\right) .
\end{aligned}
$$

The number of equations in system (8) is countable. To solve the DDE system, we apply the method of multidimensional generating functions.

We denote by $\Psi_{n}(z, t)$, where $z=\vec{z}=\left(z_{1}, z_{2}, \ldots, z_{n}\right), n$-dimensional generating function:

$$
\begin{aligned}
\Psi_{n}(z, t) & =\sum_{k_{1}=0}^{\infty} \sum_{k_{2}=0}^{\infty} \ldots \sum_{k_{n}=0}^{\infty} P\left(k_{1}, k_{2}, . ., k_{n}, t\right) z_{1}^{k_{1}} z_{2}^{k_{2}} \cdot \ldots \cdot z_{n}^{k_{n}}= \\
& =\sum_{k_{1}=1}^{\infty} \sum_{k_{2}=1}^{\infty} \ldots \sum_{k_{n}=1}^{\infty} P(k, t) \prod_{i=1}^{n} z_{i}^{k_{i}},|z|<1 .
\end{aligned}
$$

the summation is taken over each $k_{i}$, from 1 to $\infty, i=\overline{1, n}$, because the network is operating in a high load (saturation) mode.

Consider the case where the conditional probabilities $\phi_{i}(\vec{k}), \psi_{i j}(\vec{k}), \xi_{i j}(\vec{k})$, $\alpha_{i}(\vec{k}), \beta_{i j}(\vec{k}), \gamma_{i j}(\vec{k}), i, j=\overline{1, n}$ do not depend on the state of the network.

Multiply (8) by $\prod_{l=1}^{n} z_{l}^{k_{l}}$ and sum over all possible values of $k_{l}$ from 1 to $+\infty$, $l=\overline{1, n}$. Then we get: 


$$
\begin{aligned}
\sum_{k_{1}=1}^{\infty} \ldots \sum_{k_{n}=1}^{\infty} \frac{d P(\vec{k}, t)}{d t} \prod_{l=1}^{n} z_{l}^{k_{l}} & = \\
& =-\sum_{i=1}^{n}\left[\lambda_{0 i}^{+}\left(1-\phi_{i}\right)+\mu_{i}\left(1-\beta_{i i}\right)+\lambda_{0 i}^{-}\right] \sum_{k_{1}=1}^{\infty} \ldots \sum_{k_{n}=1}^{\infty} P(\vec{k}, t) \prod_{l=1}^{n} z_{l}^{k_{l}}+ \\
& +\sum_{i=1}^{n} \lambda_{0 i}^{+} \sum_{j=1}^{n} \psi_{j i} \sum_{k_{1}=1}^{\infty} \ldots \sum_{k_{n}=1}^{\infty} P\left(\vec{k}-I_{i}, t\right) \prod_{l=1}^{n} z_{l}^{k_{l}}+ \\
& +\sum_{i=1}^{n}\left[\lambda_{0 i}^{-}+\sum_{j=1}^{n} \lambda_{0 j}^{+} \xi_{j i}+\mu_{i} \alpha_{i}\right] \sum_{k_{1}=1}^{\infty} \ldots \sum_{k_{n}=1}^{\infty} P\left(\vec{k}+I_{i}, t\right) \prod_{l=1}^{n} z_{l}^{k_{l}}+ \\
& +\sum_{i, j=1}^{n} \mu_{j} \beta_{j i} \sum_{k_{1}=1}^{\infty} \ldots \sum_{k_{n}=1}^{\infty} P\left(\vec{k}+I_{j}-I_{i}, t\right) \prod_{l=1}^{n} z_{l}^{k_{l}}+ \\
& +\sum_{i \neq j}^{n} \mu_{j} \gamma_{j i} \sum_{k_{1}=1}^{\infty} \ldots \sum_{k_{n}=1}^{\infty} P\left(\vec{k}+I_{j}+I_{i}, t\right) \prod_{l=1}^{n} z_{l}^{k_{l}} .
\end{aligned}
$$

Consider the sums included in the right-hand side of relation (10). Let

$$
\sum_{1}(z, t)=\sum_{i=1}^{n} \lambda_{0 i}^{+} \sum_{j=1}^{n} \psi_{j i} \sum_{k_{1}=1}^{\infty} \ldots \sum_{k_{n}=1}^{\infty} P\left(\vec{k}-I_{i}, t\right) \prod_{l=1}^{n} z_{l}^{k_{l}},
$$

then

$$
\sum_{1}(z, t)=\sum_{i=1}^{n} \lambda_{0 i}^{+} z_{i} \sum_{\substack{\mathrm{j}=1 \\ j \neq i}}^{n} \psi_{j i} \sum_{\substack{k_{j}=0 \\ j=1, n}}^{\infty} P(\vec{k}, t) \prod_{l=1}^{n} z_{l}^{k_{l}}=\sum_{i=1}^{n} \lambda_{0 i}^{+} z_{i} \sum_{\substack{\mathrm{j}=1 \\ j \neq i}}^{n} \psi_{j i} \Psi_{n}(z, t) .
$$

For the sum

$$
\sum_{2}(z, t)=\sum_{i=1}^{n}\left[\lambda_{0 i}^{-}+\sum_{j=1}^{n} \lambda_{0 j}^{+} \xi_{j i}+\mu_{i} \alpha_{i}\right] \sum_{k_{1}=1}^{\infty} \ldots \sum_{k_{n}=1}^{\infty} P\left(\vec{k}+I_{i}, t\right) \prod_{l=1}^{n} z_{l}^{k_{l}}
$$

we have:

$$
\begin{aligned}
\sum_{2}(z, t) & =\sum_{i=1}^{n} \frac{\lambda_{0 i}^{-}+\sum_{j=1}^{n} \lambda_{0 j}^{+} \xi_{j i}+\mu_{i} \alpha_{i}}{z_{i}} \Psi_{n}(z, t)- \\
& -\sum_{i=1}^{n} \frac{\lambda_{0 i}^{-}+\sum_{j=1}^{n} \lambda_{0 j}^{+} \xi_{j i}+\mu_{i} \alpha_{i}}{z_{i}} \sum_{\substack{k_{j}=1 \\
j=1, n, j \neq i}}^{\infty} P\left(k_{1}, \ldots, k_{i-1}, 0, k_{i+1} . ., k_{n}, t\right) \prod_{\substack{l=1 \\
l \neq i}}^{n} z_{l}^{k_{l}}= \\
& =\sum_{i=1}^{n} \frac{\lambda_{0 i}^{-}+\sum_{j=1}^{n} \lambda_{0 j}^{+} \xi_{j i}+\mu_{i} \alpha_{i}}{z_{i}} \Psi_{n}(z, t)
\end{aligned}
$$


Because the QS $S_{i}$ operates in saturation mode, i.e. $P\left(k_{1}, \ldots, k_{i-1}, 0, k_{i+1} . ., k_{n}, t\right)=0$, $i=\overline{1, n}$.

For the sum

$$
\sum_{3}(z, t)=\sum_{\substack{i, j=1 \\ i \neq j}}^{n} \mu_{j} \beta_{j i} \sum_{k_{1}=1}^{\infty} \ldots \sum_{k_{n}=1}^{\infty} P\left(\vec{k}+I_{j}-I_{i}, t\right) \prod_{l=1}^{n} z_{l}^{k_{l}}
$$

we will have:

$$
\begin{aligned}
\sum_{3} & (z, t)= \\
& =\sum_{\substack{i, j=1 \\
i \neq j}}^{n} \mu_{j} \beta_{j i} \frac{z_{i}}{z_{j}} \Psi_{n}(z, t)-\sum_{\substack{i, j=1 \\
i \neq j}}^{n} \mu_{j} \beta_{j i} \frac{z_{i}}{z_{j}} \sum_{\substack{k_{m}=1 \\
m=1, n, m \neq i}}^{\infty} P\left(k_{1}, \ldots, k_{j-1}, 0, k_{j+1}, \ldots, k_{n}, t\right) \prod_{\substack{l=1 \\
l \neq i}}^{n} z_{l}^{k_{l}}= \\
& =\sum_{\substack{i, j=1 \\
i \neq j}}^{n} \mu_{j} \beta_{j i} \frac{z_{i}}{z_{j}} \Psi_{n}(z, t),
\end{aligned}
$$

for the same reasons as for the sum $\sum_{2}(z, t)$.

And finally, for the last sum

$$
\sum_{4}(z, t)=\sum_{i, j=1}^{n} \mu_{j} \gamma_{j i} \sum_{k_{1}=1}^{\infty} \ldots \sum_{k_{n}=1}^{\infty} P\left(\vec{k}+I_{j}+I_{i}, t\right) \prod_{l=1}^{n} z_{l}^{k_{l}}
$$

we will have:

$$
\begin{aligned}
& \sum_{4}(z, t)= \\
& \quad=\sum_{i, j=1}^{n} \mu_{j} \gamma_{j i} \frac{1}{z_{i} z_{j}} \Psi_{n}(z, t)-\sum_{i, j=1}^{n} \mu_{j} \gamma_{j i} \frac{1}{z_{i} z_{j}} \sum_{\substack{k_{m}=0 \\
m=1, n, m \neq j}}^{\infty} P\left(k_{1}, \ldots, k_{i-1}, 0, k_{i+1}, \ldots, k_{n}, t\right) \prod_{\substack{l=1 \\
l \neq i}}^{n} z_{l}^{k_{l}} .
\end{aligned}
$$

Since all QS of the network operate in saturation mode, then $\sum_{4}(z, t)=\sum_{i, j=1}^{n} \mu_{j} \gamma_{j i} \frac{1}{z_{i} z_{j}} \Psi_{n}(z, t)$.

Thus, for the generating function we obtain a linear homogeneous differential equation

$$
\begin{aligned}
& \frac{d \Psi_{n}(z, t)}{d t}=-\sum_{i=1}^{n}\left[\left(\lambda_{0 i}^{+}\left(1-\phi_{i}\right)+\mu_{i}\left(1-\beta_{i i}\right)+\lambda_{0 i}^{-}\right)-\right. \\
& \left.-\lambda_{0 i}^{+} z_{i} \sum_{\substack{j=1 \\
j \neq i}}^{n} \psi_{j i}-\frac{\lambda_{0 i}^{-}+\sum_{j=1}^{n} \lambda_{0 j}^{+} \xi_{j i}+\mu_{i} \alpha_{i}}{z_{i}}-\sum_{\substack{j=1 \\
i \neq j}}^{n} \mu_{j} \beta_{j i} \frac{z_{i}}{z_{j}}-\sum_{j=1}^{n} \mu_{j} \gamma_{j i} \frac{1}{z_{i} z_{j}}\right] \Psi_{n}(z, t) .
\end{aligned}
$$


Its general solution has the form

$$
\begin{aligned}
\Psi_{n}(z, t) & =C_{n} \exp \left\{-\sum_{i=1}^{n}\left[\left(\lambda_{0 i}^{+}\left(1-\phi_{i}\right)+\mu_{i}\left(1-\beta_{i i}\right)+\lambda_{0 i}^{-}\right)-\right.\right. \\
& \left.\left.-\lambda_{0 i}^{+} z_{i} \sum_{\substack{j=1 \\
j \neq i}}^{n} \psi_{j i}-\frac{\lambda_{0 i}^{-}+\sum_{j=1}^{n} \lambda_{0 j}^{+} \xi_{j i}+\mu_{i} \alpha_{i}}{z_{i}}-\sum_{\substack{j=1 \\
i \neq j}}^{n} \mu_{j} \beta_{j i} \frac{z_{i}}{z_{j}}-\sum_{j=1}^{n} \mu_{j} \gamma_{j i} \frac{1}{z_{i} z_{j}}\right] t\right\} .
\end{aligned}
$$

Assume that at the initial moment of time the network is in a state $\left(\alpha_{1}, \alpha_{2}, \ldots, \alpha_{n}, 0\right), \alpha_{i}>0, P\left(\alpha_{1}, \alpha_{2}, \ldots, \alpha_{n}, 0\right)=1, \quad P\left(k_{1}, k_{2}, \ldots, k_{n}, 0\right)=0, \quad \forall \alpha_{i} \neq k_{i}$, $i=\overline{1, n}$. Then the initial condition for equation (12) will be $\Psi_{n}(z, 0)=$ $=P\left(\alpha_{1}, \alpha_{2}, \ldots, \alpha_{n}, 0\right) \prod_{l=1}^{n} z_{l}^{\alpha_{l}}=\prod_{l=1}^{n} z_{l}^{\alpha_{l}}$. Using it, we get $C_{n}=1$.

Thus, if at the initial moment of time, the QN is in a state $\left(x_{1}, x_{2}, \ldots, x_{n}, 0\right)$, $x_{i}>0, i=\overline{1, n}$, then the expression for the generating function $\Psi_{n}(z, t)(12)$ has the form

$$
\begin{aligned}
\Psi_{n}(z, t)= & a_{0}(t) \exp \left\{\sum_{i=1}^{n} \lambda_{0 i}^{+} z_{i} \sum_{\substack{\mathrm{j}=1 \\
j \neq i}}^{n} \psi_{j i} t\right\} \exp \left\{\sum_{i=1}^{n} \frac{\lambda_{0 i}^{-}+\sum_{j=1}^{n} \lambda_{0 j}^{+} \xi_{j i}+\mu_{i} \alpha_{i}}{z_{i}} t\right\} \times \\
& \times \exp \left\{\sum_{\substack{i, j=1 \\
i \neq j}}^{n} \mu_{j} \beta_{j i} \frac{z_{i}}{z_{j}} t\right\} \exp \left\{\sum_{i, j=1}^{n} \mu_{j} \gamma_{j i} \frac{1}{z_{i} z_{j}} t\right\} \prod_{l=1}^{n} z_{l}^{\alpha_{l}},
\end{aligned}
$$

where

$$
a_{0}(t)=\exp \left\{-\sum_{i=1}^{n}\left(\lambda_{0 i}^{+}\left(1-\phi_{i}\right)+\mu_{i}\left(1-\beta_{i i}\right)+\lambda_{0 i}^{-}\right) t\right\}
$$

To find the probabilities of network states, we transform (13) to a convenient form. We expand the Maclaurin series of its exponents. Then the following statement is true. 
Theorem. The expression for the generating function is representable as

$$
\begin{aligned}
& \Psi_{n}(z, t)=a_{0}(t) \sum_{l_{1}=0}^{\infty} \ldots \sum_{l_{n}=0}^{\infty} \sum_{q_{1}=0}^{\infty} \ldots \sum_{q_{n}=0}^{\infty} \sum_{\eta_{1}=0}^{\infty} \ldots \sum_{r_{n}=0}^{\infty} \sum_{u_{1}=0}^{\infty} \ldots \sum_{u_{n}=0}^{\infty} t^{\sum_{i=1}^{n}\left(l_{i}+q_{i}+r_{i}+u_{i}\right)} \times \\
& \times \prod_{i=1}^{n}\left[\frac{\left(\lambda_{0 i}^{+}\right)^{l_{i}} \prod_{j=1}^{n} \psi_{j i}^{l_{i j}}\left(\lambda_{0 i}^{-}+\sum_{j=1}^{n} \lambda_{0 j}^{+} \xi_{j i}+\mu_{i} \alpha_{i}\right)^{q_{i}}\left(\prod_{j=1}^{n} \mu_{j} \beta_{j i}\right)^{r_{i}}\left(\prod_{j=1}^{n} \mu_{j} \gamma_{j i}\right)^{u_{i}}}{l_{i} ! q_{i} ! r_{i} ! u_{i} !} z_{i}^{\alpha_{i}+l_{i}-q_{i}+n r_{i}-R-u_{i}-U}\right],
\end{aligned}
$$

where $R=\sum_{i=1}^{n} r_{i}, U=\sum_{i=1}^{n} u_{i}$.

Proof. From the relation (13) we obtain

$$
\Psi_{n}(z, t)=a_{0}(t) a_{1}(z, t) a_{2}(z, t) a_{3}(z, t) a_{4}(z, t) \prod_{l=1}^{n} z_{l}^{\alpha_{l}},
$$

where

$$
\begin{aligned}
& a_{1}(z, t)=\exp \left\{\sum_{i=1}^{n} \lambda_{0 i}^{+} z_{i} \sum_{\substack{j=1 \\
j \neq i}}^{n} \psi_{j i} t\right\}=\prod_{i=1}^{n} \prod_{\substack{j=1 \\
j \neq i}}^{n} \exp \left\{\lambda_{0 i}^{+} z_{i} \psi_{j i} t\right\}= \\
&=\prod_{i=1}^{n} \prod_{j=1}^{n} \sum_{l_{i}=0}^{\infty} \frac{\left[\lambda_{0 i}^{+} z_{i} \psi_{j i} t\right]^{l_{i}}}{l_{i} !}=\sum_{l_{1}=0}^{\infty} \ldots \sum_{l_{n}=0}^{\infty} \prod_{i=1}^{n} \prod_{j=1}^{n} \frac{\left[\lambda_{0 i}^{+} z_{i} \psi_{j i} t\right]^{l_{i}}}{l_{i} !}= \\
&=\sum_{l_{1}=0}^{\infty} \ldots \sum_{l_{n}=0}^{\infty} \frac{t^{l_{1}+l_{2}+\ldots+l_{n}}}{l_{1} ! l_{2} ! \cdots l_{n} !} \lambda_{01}^{+l_{1}} \cdot \ldots \cdot \lambda_{0 n}^{+l_{n}} z_{1}^{l_{1}} \cdot \ldots \cdot z_{n}^{l_{n}} \prod_{j=1}^{n} \psi_{j 1}^{l_{1}} \cdots \psi_{j n}^{l_{n}}, \\
& a_{2}(z, t)=\exp \left\{\sum_{i=1}^{n} \frac{\lambda_{0 i}^{-}+\sum_{j=1}^{n} \lambda_{0 j}^{+} \xi_{j i}+\mu_{i} \alpha_{i}}{z_{i}} t=\prod_{i=1}^{n} \exp \left\{\frac{\lambda_{0 i}^{-}+\sum_{j=1}^{n} \lambda_{0 j}^{+} \xi_{j i}+\mu_{i} \alpha_{i}}{z_{i}} t=\right.\right. \\
&=\prod_{i=1}^{n} \sum_{q_{i}=0}^{\infty} \frac{\left[\left(\lambda_{0 i}^{-}+\sum_{j=1}^{n} \lambda_{0 j}^{+} \xi_{j i}+\mu_{i} \alpha_{i}\right) z_{i}^{-1} t\right]^{q_{i}}}{q_{i} !}= \\
&=\sum_{q_{1}=0}^{\infty} \ldots \sum_{q_{n}=0}^{\infty} t^{q_{1}} \ldots t^{q_{n}} \frac{\left(\lambda_{01}^{-}+\sum_{j=1}^{n} \lambda_{0 j}^{+} \xi_{j 1}+\mu_{1} \alpha_{1}\right)^{q_{1}} \cdot \ldots \cdot\left(\lambda_{0 n}^{-}+\sum_{j=1}^{n} \lambda_{0 j}^{+} \xi_{j n}+\mu_{n} \alpha_{n}\right)^{q_{n}}}{q_{1} ! \ldots \cdot q_{n} !} z_{1}^{-q_{1}} \cdots z_{n}^{-q_{n}}
\end{aligned}
$$




$$
\begin{aligned}
& a_{3}(z, t)=\exp \left\{\sum_{\substack{i, j=1 \\
i \neq j}}^{n} \mu_{j} \beta_{j i} \frac{z_{i}}{z_{j}} t\right\}=\prod_{i=1}^{n} \prod_{\substack{j=1 \\
i \neq j}}^{n} \exp \left\{\mu_{j} \beta_{j i} \frac{z_{i}}{z_{j}} t\right\}= \\
& =\prod_{i=1}^{n} \prod_{\substack{j=1 \\
j \neq i}}^{n} \sum_{r_{i}=0}^{\infty} \frac{\left[\mu_{j} \beta_{j i} z_{j}^{-1} z_{i} t\right]^{r_{i}}}{r_{i} !}=\sum_{r_{1}=0}^{\infty} . \cdot \sum_{r_{n}=0}^{\infty} \prod_{i=1}^{n} \prod_{\substack{j=1 \\
j \neq i}}^{n} \frac{\left[\mu_{j} \beta_{j i} z_{j}^{-1} z_{i} t\right]^{r_{i}}}{r_{i} !}= \\
& =\sum_{\eta_{1}=0}^{\infty} \ldots \sum_{r_{n}=0}^{\infty} t^{\eta_{1}} \cdots t^{r_{n}} \frac{\left(\prod_{j=1}^{n} \mu_{j} \beta_{j 1}\right)^{r_{1}} \cdot \ldots \cdot\left(\prod_{j=1}^{n} \mu_{j} \beta_{j n}\right)^{r_{n}}}{r_{1} ! \cdots r_{n} !} \\
& z_{1}^{-r_{1}-r_{2}-\ldots-r_{n}} z_{2}^{-r_{1}-r_{2}-\ldots-r_{n}} \cdots z_{n}^{-r_{1}-r_{2}-\ldots-r_{n}} z_{1}^{n r_{1}} z_{2}^{n r_{2}} \ldots z_{n}^{n r_{n}}= \\
& =\sum_{n_{1}=0}^{\infty} \ldots \sum_{r_{n}=0}^{\infty} t^{r_{1}} \cdots t^{r_{n}} \frac{\left(\prod_{j=1}^{n} \mu_{j} \beta_{j 1}\right)^{r_{1}} \cdot \ldots \cdot\left(\prod_{j=1}^{n} \mu_{j} \beta_{j n}\right)^{r_{n}}}{r_{1} ! \cdots r_{n} !} z_{1}^{n r_{1}-R} \cdots z_{n}^{n r_{n}-R}, R=\sum_{i=1}^{n} r_{i}, \\
& a_{4}(z, t)=\exp \left\{\sum_{i, j=1}^{n} \mu_{j} \gamma_{j i} \frac{1}{z_{i} z_{j}} t\right\}=\sum_{u_{1}=0}^{\infty} . \sum_{u_{n}=0}^{\infty} \prod_{i=1}^{n} \prod_{j=1}^{n} \frac{\left[\mu_{j} \gamma_{j i} t z_{i}^{-1} z_{j}^{-1}\right]^{u_{i}}}{u_{i} !}= \\
& =\sum_{u_{1}=0}^{\infty} \ldots \sum_{u_{n}=0}^{\infty} t^{u_{1}+\ldots+u_{n}} \frac{\left(\prod_{j=1}^{n} \mu_{j} \gamma_{j 1}\right)^{u_{1}} \cdot \ldots \cdot\left(\prod_{j=1}^{n} \mu_{j} \gamma_{j n}\right)^{u_{n}}}{u_{1} ! \ldots \cdot u_{n} !} z_{1}^{-u_{1}-u_{2}-\ldots-u_{n}} \ldots z_{n}^{-u_{1}-u_{2}-\ldots-u_{n}} z_{1}^{-u_{1}} \ldots z_{n}^{-u_{n}}= \\
& =\sum_{u_{1}=0}^{\infty} \ldots \sum_{u_{n}=0}^{\infty} t^{u_{1}+\ldots+u_{n}} \frac{\left(\prod_{j=1}^{n} \mu_{j} \gamma_{j 1}\right)^{u_{1}} \cdot \ldots \cdot\left(\prod_{j=1}^{n} \mu_{j} \gamma_{j n}\right)^{u_{n}}}{u_{1} ! \ldots \cdot u_{n} !} z_{1}^{-\left(U+u_{1}\right)} \cdot \ldots \cdot z_{n}^{-\left(U+u_{n}\right)}, U=\sum_{i=1}^{n} u_{i} .
\end{aligned}
$$

Multiplying $a_{0}(t), a_{1}(z, t), a_{2}(z, t), a_{3}(z, t), a_{4}(z, t)$ and $\prod_{l=1}^{n} z_{l}^{\alpha_{l}}$ we obtain an expression (14). The theorem is proved.

With the obtained expression for the generating function, state probabilities of the considered network can be found. The state probability is the coefficient at $z_{1}^{k_{1}} z_{2}^{k_{2}}, \ldots, z_{n}^{k_{n}}$ in the expansion of (9) in multiple series (15), on condition, that at the initial time the network is in a state $\left(\alpha_{1}, \alpha_{2}, \ldots, \alpha_{n}, 0\right)$. 
Example 1. Consider the network consisting of $n=4 \mathrm{CMO}$. The intensities of the receipt of positive and negative customers in each QS and also the intensities of service are equal to respectively $\lambda_{01}^{+}=17 ; \lambda_{01}^{-}=9 ; \lambda_{02}^{+}=13 ; \lambda_{02}^{-}=5 ; \lambda_{03}^{+}=11 ; \lambda_{03}^{-}=3$; $\lambda_{04}^{+}=8 ; \lambda_{04}^{-}=1 ; \mu_{i}=50, i=1,5$. Customers in the QS joins the queue with probability $f^{(1)}=0,1 ; f^{(2)}=0,2 ; f^{(3)}=0,15 ; f^{(4)}=0,25$. The probabilities of transferring customers from one system to another are equal to $p_{i 0}=0,2, p_{i j}^{+}=p_{i j}^{-}=0,1$, $i, j=\overline{1,4}$. Solving systems (1)-(6) in the Wolfram package, we find conditional probabilities $\phi_{1}=0,269 ; \phi_{2}=0,239 ; \phi_{3}=0,254 ; \phi_{4}=0,224 ; \psi_{i 1}=0,67 ; \psi_{i 2}=0,174$; $\psi_{i 3}=0,174 ; \quad \psi_{i 4}=0,165, \quad i=\overline{1,4}, \quad \xi_{i 1}=0,73, \quad \xi_{i 2}=0,14, \quad \xi_{i 3}=0,1, \quad \xi_{i 4}=0,03$, $\alpha_{i}=0,2986, \beta_{1 i}=0,268 ; \beta_{2 i}=0,07 ; \beta_{3 i}=0,07 ; \beta_{4 i}=0,065 ; \gamma_{i 1}=0,368 ; \gamma_{i 2}=0,17$; $\gamma_{i 3}=0,17 ; \gamma_{i 4}=0,166$; Finding the state probability $(1,2,3,4)$ provided that the state $(1,1,1,1)$ was initial. It is the coefficient of $z_{1}^{1} z_{2}^{2} z_{3}^{3} z_{4}^{4}$ in the expansion of the generating function in a series $k_{c}=1+l_{c}-q_{c}+n r_{c}-R-u_{c}-U, u_{c}+U=1+l_{c}-q_{c}+n r_{c}-R-k_{c}$. Figure 1 shows a chart of the probability of this state in the time interval $[0 ; 10]$.

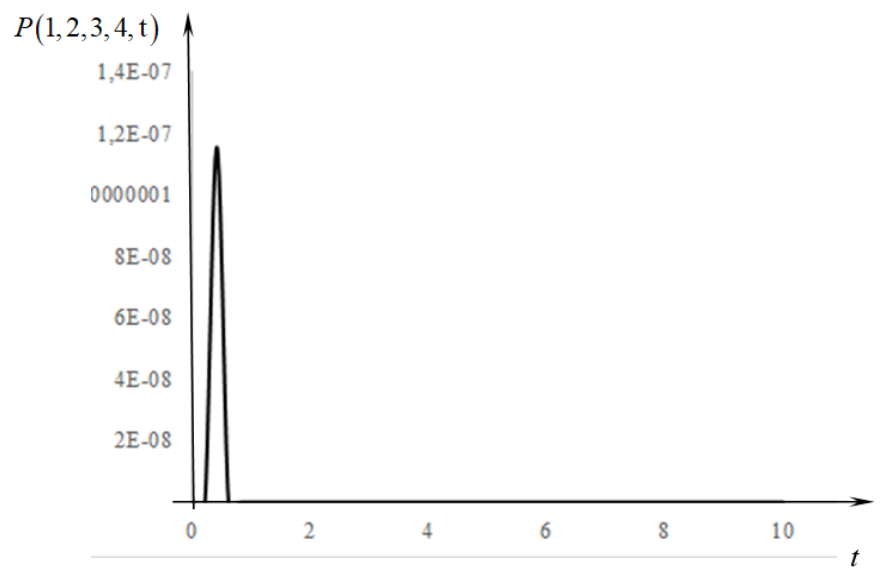

Fig. 1 . State probability $(1,2,3,4)$ on $[0 ; 10]$

\section{References}

[1] Gelenbe, E. (1991). Product-form queueing networks with negative and positive customers. Journal of Applied Probability, 28, 656-663.

[2] Gelenbe, E., \& Schassberger, R. (1992). Stability of product-form G-networks. Probability in Engineering and Informational Science, 6, 271-276.

[3] Naumenko, V., \& Matalytski, M. (2013). Analysis networks with positive and negative customers in transition mode. Tomsk State University Journal. Management, Computer Engineering and Informatics, 4(25), 61-70. 
[4] Malinkovski, V. (1991). Queuing networks with bypasses Nodes. Automation and Remote Control, 2, 102-110.

[5] Matalytski, M., \& Naumenko, V. (2013). Network analysis with bypasses of queueing systems by multiple claims. Grodno State University Journal, 2(1), 152-159.

[6] Malinkovski, Y., \& Nikitenko, O. (2000). Stationary distribution of network states with bypasses and negative claims. Automation and Remote Control, 8, 79-85.

[7] Dovzhenok, T. (2002). Invariance of the stationary distribution of networks with bypasses and "negative" claims. Automation and Remote Control, 9, 97-110.

[8] Kopats, D., \& Matalytski, M. (2019). Finding the expected revenues in the G-network with bypasses. Information Technologies and Mathematical Modeling: Proc. of the XVI Int. Conf., Tomsk, June 26-30, 2019, 189-195. 